\title{
A PROBLEM OF RANKIN ON SETS WITHOUT GEOMETRIC PROGRESSIONS
}

\author{
MELVYN B. NATHANSON AND KEVIN O'BRYANT
}

\begin{abstract}
A geometric progression of length $k$ and integer ratio is a set of numbers of the form $\left\{a, a r, \ldots, a r^{k-1}\right\}$ for some positive real number $a$ and integer $r \geq 2$. For each integer $k \geq 3$, a greedy algorithm is used to construct a strictly decreasing sequence $\left(a_{i}\right)_{i=1}^{\infty}$ of positive real numbers with $a_{1}=1$ such that the set

$$
G^{(k)}=\bigcup_{i=1}^{\infty}\left(a_{2 i}, a_{2 i-1}\right]
$$

contains no geometric progression of length $k$ and integer ratio. Moreover, $G^{(k)}$ is a maximal subset of $(0,1]$ that contains no geometric progression of length $k$ and integer ratio. It is also proved that there is a strictly increasing sequence $\left(A_{i}\right)_{i=1}^{\infty}$ of positive integers with $A_{1}=1$ such that $a_{i}=1 / A_{i}$ for all $i=1,2,3, \ldots$

The set $G^{(k)}$ gives a new lower bound for the maximum cardinality of a subset of the set of integers $\{1,2, \ldots, n\}$ that contains no geometric progression of length $k$ and integer ratio.
\end{abstract}

\section{REAL AND INTEGRAL GEOMETRIC PROGRESSIONS}

Let $\mathbf{R}$ denote the real numbers. For $t \in \mathbf{R}$, let $\mathbf{R}_{>t}$ denote the set of all real numbers $x>t$. Let $[x]$ denote the integer part of the real number $x$. For real numbers $u<v$, we define the intervals

$$
(u, v]=\{x \in \mathbf{R}: u<x \leq v\} \quad \text { and } \quad[u, v)=\{x \in \mathbf{R}: u \leq x<v\} .
$$

Let $X$ be a set of positive real numbers, and let $u, v \in \mathbf{R}_{>0}$ with $u<v$. The dilation of the set $X$ by $q \in \mathbf{R}_{>0}$ is the set

$$
q * X=\{q x: x \in X\} .
$$

The reciprocal of the set $X$ is the set

$$
X^{-1}=\left\{x^{-1}: x \in X\right\} .
$$

For example, $q *(u, v]=(q u, q v]$ and $(1 / v, 1 / u]^{-1}=[u, v)$.

If $A=\left(a_{0}, a_{1}, \ldots, a_{k-1}\right)$ is a finite sequence of positive real numbers, then the dilation of the sequence $A$ by $q$ is the sequence $q * A=\left(q a_{0}, q a_{1}, \ldots, q a_{k-1}\right)$ and the reciprocal of $A$ is the sequence $A^{-1}=\left(1 / a_{0}, 1 / a_{1}, \ldots, 1 / a_{k-1}\right)$.

Let $\mathbf{N}$ denote the set of positive integers, and let $\mathbf{N}^{\sharp}=\mathbf{N} \backslash\{1\}$ denote the set of all integers $r>1$. Let $k \in \mathbf{N}$ and let $r, a \in \mathbf{R}_{>0}$. A geometric progression of length $k$ and ratio $r$ with first term $a$ is a sequence of the form

$$
\left(a, a r, a r^{2}, \ldots, a r^{k-1}\right)=a *\left(1, r, r^{2}, \ldots, r^{k-1}\right) .
$$

Date: October 1, 2018.

2010 Mathematics Subject Classification. 11B05 11B25, 11B75, 11B83, $05 \mathrm{D} 10$.

Key words and phrases. Geometric progression-free sequences, Ramsey theory. 
This is an integer geometric progression of length $k$ if $a r^{j} \in \mathbf{N}$ for all $j \in\{0,1, \ldots, k-$ $1\}$. If $\left(a, a r, a r^{2}, \ldots, a r^{k-1}\right)$ is an integer geometric progression, then the ratio $r$ must be a rational number. For example, $(8,12,18,27)$ is an integer geometric progression of length 4 with ratio $3 / 2$.

Note that the dilation by a positive real number $q$ of the geometric progression $\left(a, a r, a r^{2}, \ldots, a r^{k-1}\right)$ of length $k$, ratio $r$, and first term $a$ is the geometric progression $\left(q a, q a r, q a r^{2}, \ldots, q a r^{k-1}\right)$ of length $k$, ratio $r$, and first term qa. The reciprocal of the geometric progression $\left(a, a r, a r^{2}, \ldots, a r^{k-1}\right)$ is the geometric progression

$$
\left(\frac{1}{a}, \frac{1}{a}\left(\frac{1}{r}\right), \frac{1}{a}\left(\frac{1}{r}\right)^{2}, \ldots, \frac{1}{a}\left(\frac{1}{r}\right)^{k-1}\right)
$$

of length $k$, ratio $1 / r$, and first term $1 / a$.

The reverse of the sequence $\left(a_{1}, a_{2}, \ldots, a_{k-1}, a_{k}\right)$ is the sequence $\left(a_{k}, a_{k-1}, \ldots, a_{2}, a_{1}\right)$. The reverse of the reciprocal of the geometric progression $\left(a, a r, a r^{2}, \ldots, a r^{k-1}\right)$ is the geometric progression $\left(b, b r, b r^{2}, \ldots, b r^{k-1}\right)$, where $b=1 /\left(a r^{k-1}\right)$.

Thus, a set $G$ of real numbers contains no geometric progression of length $k$ if and only if the dilation $q * G$ contains no geometric progression of length $k$ for every positive real number $q$. Moreover, if a set $G$ contains no geometric progression of length $k$, then no subset of $G$ contains a geometric progression of length $k$. It follows that if a set $G$ contains no geometric progression of length $k$, then, for every positive real number $q$, the set $(q * G) \cap \mathbf{N}$ is a set of positive integers that contains no geometric progression of length $k$. Similarly, if $G$ contains no geometric progression of length $k$, then the set of $G^{-1} \cap \mathbf{N}$ is a set of positive integers that contains no geometric progression of length $k$.

A geometric progression of length $k$ with integer ratio is a geometric progression of length $k$ with ratio $r \in \mathbf{N}^{\sharp}$. An integer geometric progression of length $k$ with integer ratio is a geometric progression of the form $\left(a, a r, a r^{2}, \ldots, a r^{k-1}\right)$ with $a \in \mathbf{N}$ and $r \in \mathbf{N}^{\sharp}$.

For positive integers $k$ and $n$, let $g_{k}(n)$ denote the cardinality of the largest subset of the set $\{1,2,3, \ldots, n\}$ that contains no integer geometric progression of length $k$ with integer ratio, and let $\hat{g}_{k}(n)$ denote the cardinality of the largest subset of the set $\{1,2,3, \ldots, n\}$ that contains no integer geometric progression of length $k$ with rational ratio.

We have $g_{1}(n)=\hat{g}_{1}(n)=0$ for all $n \in \mathbf{N}$, and $g_{k}(n)=\hat{g}_{k}(n)=n$ if $n<k$. Moreover, $\hat{g}_{2}(n)=1$ for $n \geq 2$. We compute $g_{2}(n)$ in the next section. In this paper we obtain new lower bounds for the function $g_{k}(n)$ for $k \geq 3$.

For every integer $k \geq 3$, there are four basic unsolved problems:

(1) Determine the cardinality and the structure of the maximal subsets of $\{1,2, \ldots, n\}$ that contain no geometric progression of length $k$ with integer ratio. In particular, what is the maximum cardinality $g_{k}(n) ?$

(2) Determine the cardinality and the structure of the maximal subsets of $\{1,2, \ldots, n\}$ that contain no geometric progression of length $k$ with rational ratio. What is the maximum cardinality $\hat{g}_{k}(n)$ ?

(3) Determine the density and structure of maximal infinite sets of positive integers that contain no geometric progression of length $k$ with integer ratio. What is the least upper bound of the densities of such sets? Is this least upper bound achieved? 
(4) Determine the density and structure of maximal infinite sets of positive integers that contain no geometric progression of length $k$ with rational ratio. What is the least upper bound of the densities of such sets? Is this least upper bound achieved?

Very little is known about these problems. The literature consists mostly of lower bounds for the maximum cardinalities in Problems 1 and 2, and for the densities in Problems 3 and 4. In this paper we improve the lower bounds in Problem 1. Our method is to use a greedy algorithm to construct, for every integer $k \geq 3$, a unique maximal subset of the unit interval $(0,1]$ that contains no geometric progression of length $k$ with integer ratio, and to use the measure of this set to obtain new lower bounds for the finite sets considered in Problem 1.

The earliest discussion of sets with no $k$-term geometric progression is in a paper of Rankin [6] in 1960 that was concerned with sets of integers containing no $k$-term arithmetic progression.

\section{INTEGRAL GEOMETRIC PROGRESSIONS OF LENGTH 2}

We can quickly solve the problem of integer geometric progressions of length 2 with integer ratio. Every set $\{a, b\}$ of positive real numbers with $a<b$ is a geometric progression of length 2 with ratio $r=b / a$. In particular, every set $\{a, b\}$ of positive integers with $a<b$ is a geometric progression of length 2 with rational ratio $r=b / a$. The set $\{a, b\}$ is an integer geometric progression of length 2 with integer ratio if and only if $a, b \in \mathbf{N}$ and $a$ divides $b$. Thus, a set $S$ of positive integers contains no 2-term geometric progression if and only if $S$ is primitive in the sense that no element of $S$ divides another element of $S$.

The following is a classical result in combinatorial number theory.

Theorem 1. Let $g_{2}(n)$ denote the cardinality of the largest primitive subset of $\{1,2, \ldots, n\}$, that is, the largest subset of $\{1,2, \ldots, n\}$ that contains no integer geometric progression of length 2 with integer ratio. Then $g_{2}(n)=\left[\frac{n+1}{2}\right]$.

Proof. For every positive integer $n$, the interval

$$
S=\left(\left[\frac{n}{2}\right], n\right]=\left\{\left[\frac{n}{2}\right]+1,\left[\frac{n}{2}\right]+2, \ldots, n-1, n\right\}
$$

is primitive because $2\left(\left[\frac{n}{2}\right]+1\right) \geq n+1>n$. The cardinality of this set is $\left[\frac{n+1}{2}\right]$, and so $g_{2}(n) \geq\left[\frac{n+1}{2}\right]$.

Let $S$ be any primitive subset of $\{1,2, \ldots, n\}$. Each element $s \in S$ can be written uniquely in the form $s=2^{k(s)} a(s)$, where $k(s)$ is a nonnegative integer and $a(s)$ is an odd integer in $\{1,2, \ldots, n\}$. If $a\left(s_{1}\right)=a\left(s_{2}\right)$ for integers $s_{1}, s_{2} \in S$ with $s_{1}<s_{2}$, then $s_{1}$ divides $s_{2}$. It follows that the cardinality of the primitive set $S$ is at most the number of odd integers in $\{1,2, \ldots, n\}$, and so $g_{2}(n) \leq\left[\frac{n+1}{2}\right]$. This completes the proof.

\section{GoOd SET, BAD SET}

Let $k$ be an integer, $k \geq 3$. A $k$-good set is a set of positive real numbers that contains no geometric progression of length $k$ with integer ratio. For example, the set

$$
G_{1}^{(k)}=\left(\frac{1}{2^{k-1}}, 1\right]
$$


is $k$-good because, if $x \in G_{1}^{(k)}$ and $r \in \mathbf{N}^{\sharp}$, then $x r^{k-1} \geq x 2^{k-1}>1$ and so $\left\{x, x r, x r^{2}, \ldots, x r^{k-1}\right\}$ is not a subset of $G_{1}^{(k)}$.

Let $G$ be a $k$-good subset of $(0,1]$, and let $x \in(0,1] \backslash G$. The real number $x$ is $k$-bad with respect to $G$ if there exists an integer $r \in \mathbf{N}^{\sharp}$ such that $G \cup\{x\}$ contains the $k$-term geometric progression $\left(x, x r, x r^{2}, \ldots, x r^{k-1}\right)$. Thus, if $x$ is $k$-bad with respect to $G$, then the set $G \cup\{x\}$ is not $k$-good.

For example, the number $1 / 2^{k}$ is $k$-bad with respect to the $k$-good set $G_{1}^{(k)}$ because $\left(1 / 2^{k}, 1 / 2^{k-1}, 1 / 2^{k-2}, \ldots, 1 / 2,1\right)$ is a $k$-term geometric progression with ratio $r=2$ contained in $G_{1}^{(k)} \cup\left\{1 / 2^{k}\right\}$.

The number $3 / 16$ is 3 -bad with respect to the 3 -good set $G_{1}^{(3)}=(1 / 4,1]$ because, with $r=2$,

$$
\left\{\frac{3}{16} r, \frac{3}{16} r^{2}\right\}=\left\{\frac{3}{8}, \frac{3}{4}\right\} \subseteq\left(\frac{1}{4}, 1\right]=G_{1}^{(3)}
$$

and so the $\operatorname{set} G_{1}^{(3)} \cup\{3 / 16\}$ contains the 3 -term geometric progression (3/16,3/8,3/4). Similarly, $1 / 10$ is 3 -bad with respect to $G_{1}^{(3)}$ because, with $r=3$,

$$
\left\{\frac{1}{10}, \frac{1}{10} r, \frac{1}{10} r^{2}\right\}=\left\{\frac{1}{10}, \frac{3}{10}, \frac{9}{10}\right\} \subseteq G_{1}^{(3)} \cup\left\{\frac{1}{10}\right\} .
$$

Note that if $G$ is a $k$-good subset of $(0,1]$ and if $x \in(0,1] \backslash G$ is $k$-bad with respect to $G$, then $x$ is also $k$-bad with respect to the good set $G \cap(x, 1]$, because $x<r^{j} x$ for all $r \in \mathbf{N}^{\sharp}$ and $j \in\{1, \ldots, k-1\}$.

The real number $x \in(0,1] \backslash G$ is $k$-good with respect to $G$ if $x$ is not $k$-bad with respect to $G$. Thus, $x$ is $k$-good with respect to $G$ if and only if, for every $r \in \mathbf{N}^{\sharp}$, there exists $j \in\{1,2, \ldots, k-1\}$ such that $x r^{j} \notin G$. Because $G \subseteq(0,1]$ and $x r^{k-1} \notin G$ if $r>(1 / x)^{1 /(k-1)}$, it follows that $x \in[0,1) \backslash G$ is $k$-good with respect to $G$ if and only if, for every integer $r$ with $2 \leq r \leq(1 / x)^{1 /(k-1)}$, there exists $j \in\{1,2, \ldots, k-1\}$ with $x r^{j} \notin G$.

For every $k$-good set $G \subseteq(0,1]$, we define

$$
\operatorname{Bad}(G)=\{x \in(0,1] \backslash G: x \text { is } k \text {-bad with respect to } G\} .
$$

Thus, $G \cup\{x\}$ is $k$-good for all $x \in(0,1] \backslash(G \cup \operatorname{Bad}(G))$. If $G$ and $G^{\prime}$ are $k$-good sets with $G \subseteq G^{\prime}$, then $\operatorname{Bad}(G) \subseteq \operatorname{Bad}\left(G^{\prime}\right)$.

For fixed $k$, we usually write "good" instead of " $k$-good" and "bad" instead of "k-bad."

\section{Construction of a Good Set of Real numbers}

Fix the integer $k \geq 3$. We shall use a greedy algorithm to construct a large good set contained in the interval $(0,1]$. We begin with some simple observations about good and bad sets.

Lemma 1. Let $k \geq 3$, let $0<a<1$, and let $\delta_{k}(a)=a^{(k-1) /(k-2)}$.

(i) For every $\delta>0$, the interval $(0, \delta]$ is not good.

(ii) Every number in the interval $\left(0, a^{2}\right]$ is good with respect to the interval $(a, 1]$.

(iii) Let $x \in(0,1]$. If $x r^{j} \in(a, 1]$ for some $r \in \mathbf{N}^{\sharp}$ and all $j \in\{1, \ldots, k-1\}$, then $x>\delta_{k}(a)$.

(iv) If $G$ is a good set with $G \subseteq(a, 1]$, then $\left(0, \delta_{k}(a)\right] \cap \operatorname{Bad}(G)=\emptyset$.

Note that $0<\delta_{k}(a)<a$. 
Proof. (i) We have $0<2^{1-k} \delta<\delta$. For every $x \in\left(0,2^{1-k} \delta\right]$, we have

$$
0<x<2 x<\cdots<2^{k-1} x \leq 2^{k-1} 2^{1-k} \delta=\delta
$$

and so $(0, \delta]$ contains the $k$-term geometric progression $\left\{x, 2 x, \ldots, 2^{k-1} x\right\}$. Thus, the interval $(0, \delta]$ is not good.

(ii) If $x \in\left(0, a^{2}\right], r \in \mathbf{N}^{\sharp}$ and $x r \in(a, 1]$, then $x r>a$ and $r>a / x$. It follows that

$$
x r^{k-1} \geq x r^{2}>x\left(\frac{a}{x}\right)^{2}=\frac{a^{2}}{x}>1
$$

and so $x$ is good with respect to $(a, 1]$.

$x$ is bad with respect to $(a, 1]$, then there exists $r \in \mathbf{N}^{\sharp}$ such that $x r^{i} \in(a, 1]$

(iii) If $r \in \mathbf{N}^{\sharp}$ and

$$
a<r x<\cdots<r^{k-1} x \leq 1
$$

then

$$
\frac{a}{r}<x \leq \frac{1}{r^{k-1}}
$$

and so $1 / r>a^{1 /(k-2)}$. Therefore,

$$
x>\frac{a}{r}>a a^{1 /(k-2)}=a^{(k-1) /(k-2)}=\delta_{k}(a) .
$$

(iv) This follows immediately from (iii).

Lemma 2. Let $\left(a_{i}\right)_{i=1}^{2 n}$ be a strictly decreasing sequence of positive real numbers with $a_{1} \leq 1$ such that

$$
G_{n}=\bigcup_{i=1}^{n}\left(a_{2 i}, a_{2 i-1}\right]
$$

is a good set. If $x \in \operatorname{Bad}\left(G_{n}\right)$, then there exists $\delta>0$ such that $(x-\delta, x] \subseteq \operatorname{Bad}\left(G_{n}\right)$.

Proof. We have $G_{n} \subseteq(0,1]$. If $x \in \operatorname{Bad}\left(G_{n}\right)$, then there exists $r \in \mathbf{N}^{\sharp}$ such that $x r^{j} \in G_{n}$ for all $j \in\{1, \ldots, k-1\}$. It follows that, for each $j \in\{1, \ldots, k-1\}$, there exists $i_{j} \in\{1, \ldots, n\}$ such that

$$
x r^{j} \in\left(a_{2 i_{j}}, a_{2 i_{j}-1}\right]
$$

or, equivalently,

$$
\frac{a_{2 i_{j}}}{r^{j}}<x \leq \frac{a_{2 i_{j}-1}}{r^{j}} .
$$

Choose $\delta>0$ such that

$$
\frac{a_{2 i_{j}}}{r^{j}}<x-\delta<x \leq \frac{a_{2 i_{j}-1}}{r^{j}}
$$

for all $j \in\{1, \ldots, k-1\}$. If $y \in(x-\delta, x]$, then

$$
a_{2 i_{j}}<(x-\delta) r^{j}<y r^{j} \leq x r^{j} \leq a_{2 i_{j}-1}
$$

and so $y r^{j} \in\left(a_{2 i_{j}}, a_{2 i_{j}-1}\right] \subseteq G_{n}$ for all $j \in\{1, \ldots, k-1\}$. Thus, $(x-\delta, x] \subseteq$ $\operatorname{Bad}\left(G_{n}\right)$.

Lemma 3. Let $\left(a_{i}\right)_{i=1}^{2 n+1}$ be a strictly decreasing sequence of positive real numbers with $a_{1} \leq 1$ such that

$$
G_{n}=\bigcup_{i=1}^{n}\left(a_{2 i}, a_{2 i-1}\right]
$$


is a good set, and

$$
\bigcup_{i=1}^{n}\left(a_{2 i+1}, a_{2 i}\right] \subseteq \operatorname{Bad}\left(G_{n}\right) .
$$

If $x \in\left(a_{2 n+1} / 2, a_{2 n+1}\right]$ is good with respect to $G_{n}$, then there exists $\delta>0$ such that $(x-\delta, x] \cup G_{n}$ is good.

Proof. Let $x \in\left(a_{2 n+1} / 2, a_{2 n+1}\right]$ be good with respect to $G_{n}$. For each $r \in \mathbf{N}^{\sharp}$ there exists $j_{r} \in\{1, \ldots, k-1\}$ such that $x r^{j_{r}} \notin G_{n}$. Let $r_{0}$ be the smallest integer such that $r_{0} \geq 2$ and $x r_{0}^{k-1}>a_{1}$. Then $x>a_{1} / r_{0}^{k-1}$, and there exists $\delta_{0}>0$ such that $x-\delta_{0}>a_{1} / r_{0}^{k-1}$. If $y \in\left(x-\delta_{0}, x\right]$ and $r \geq r_{0}$, then

$$
y r^{k-1}>\left(x-\delta_{0}\right) r_{0}^{k-1}>a_{1}
$$

and so $y r^{k-1} \notin G_{n}$.

For each integer $r$ such that $2 \leq r<r_{0}$, we have

$$
a_{2 n+1}<2 x \leq x r^{j_{r}} \leq x r^{k-1} \leq a_{1}
$$

and so there exists $i_{r} \in\{1, \ldots, n\}$ such that

$$
a_{2 i_{r}+1}<x r^{j_{r}} \leq a_{2 i_{r}} .
$$

Equivalently,

$$
\frac{a_{2 i_{r}+1}}{r^{j_{r}}}<x \leq \frac{a_{2 i_{r}}}{r^{j_{r}}} .
$$

Choose $0<\delta_{1}<x / 2$ such that

$$
\frac{a_{2 i_{r}+1}}{r^{j_{r}}}<x-\delta_{1}<x \leq \frac{a_{2 i_{r}}}{r^{j_{r}}}
$$

for all $r \in \mathbf{N}^{\sharp}$ with $r<r_{0}$. If $y \in\left(x-\delta_{1}, x\right]$ and $r<r_{0}$, then

$$
a_{2 i_{r}+1}<\left(x-\delta_{1}\right) r^{j_{r}}<y r^{j_{r}} \leq x r^{j_{r}} \leq a_{2 i_{r}}
$$

and so $y r^{j_{r}} \notin G_{n}$. Let $\delta=\min \left(\delta_{0}, \delta_{1}\right)$. It follows that if $y \in(x-\delta, x]$, then $y$ is good with respect to $G_{n}$. This completes the proof.

Theorem 2. Let $k \geq 3$. There exists a unique strictly decreasing sequence $\left(a_{i}\right)_{i=1}^{\infty}$ of positive real numbers with $a_{1}=1$ such that

$$
G=\bigcup_{i=1}^{\infty}\left(a_{2 i}, a_{2 i-1}\right]
$$

is a good set, and

$$
\operatorname{Bad}(G)=\bigcup_{i=1}^{\infty}\left(a_{2 i+1}, a_{2 i}\right] .
$$

Proof. We construct the sequence $\left(a_{i}\right)_{i=1}^{\infty}$ by induction.

Let $a_{1}=1$. If $x>2^{1-k}$, then for all $r \in \mathbf{N}^{\sharp}$ we have

$$
r^{k-1} x>2^{k-1} 2^{1-k}=1
$$

and so $\left(2^{1-k}, 1\right]$ is a good set. Therefore,

$$
a_{2}=\inf \left\{x \in(0,1]:\left(x, a_{1}\right] \text { is } \operatorname{good}\right\} \leq 2^{1-k} .
$$

We observe that $\left[2^{1-k}, 1\right]$ is not a good set because, with $y=2^{1-k}$, we have $\left\{y, y 2, \ldots, y 2^{k-1}\right\} \subseteq\left[2^{1-k}, 1\right]$. Therefore,

$$
a_{2}=\frac{1}{2^{k-1}} \in \operatorname{Bad}\left(G_{1}\right)
$$


where

We define

$$
G_{1}=\left(a_{2}, a_{1}\right]=\left(\frac{1}{2^{k-1}}, 1\right]
$$

$$
a_{3}=\inf \left\{x \in(0,1]:\left(x, a_{2}\right] \subseteq \operatorname{Bad}\left(G_{1}\right)\right\} .
$$

It follows from Lemmas 1 and 2 that $0<\delta_{k}\left(a_{2}\right) \leq a_{3}<a_{2}$ and $a_{3} \notin \operatorname{Bad}\left(G_{1}\right)$.

Let $n \geq 1$, and assume that there is a unique strictly decreasing sequence $\left(a_{i}\right)_{i=1}^{2 n+1}$ of positive real numbers with $a_{1}=1$ such that

$$
G_{n}=\bigcup_{i=1}^{n}\left(a_{2 i}, a_{2 i-1}\right]
$$

is a good set,

$$
\bigcup_{i=1}^{n}\left(a_{2 i+1}, a_{2 i}\right] \subseteq \operatorname{Bad}\left(G_{n}\right) .
$$

and

$$
a_{2 n+1}=\inf \left\{x \in(0,1]:\left(x, a_{2 n}\right] \subseteq \operatorname{Bad}\left(G_{n}\right)\right\} .
$$

By Lemma 2, the number $a_{2 n+1}$ is good with respect to $G_{n}$. Let

$$
a_{2 n+2}=\inf \left\{x \in\left(0, a_{2 n+1}\right]:\left(x, a_{2 n+1}\right] \text { is good with respect to } G_{n}\right\} \text {. }
$$

Let

$$
G_{n+1}=G_{n} \cup\left(a_{2 n+2}, a_{2 n+1}\right] .
$$

Lemmas 1 and 3 imply that $0<a_{2 n+2}<a_{2 n+1}$, and that $a_{2 n+2} \in \operatorname{Bad}\left(G_{n+1}\right)$. We define

$$
a_{2 n+3}=\inf \left\{x \in(0,1]:\left(x, a_{2 n+2}\right] \subseteq \operatorname{Bad}\left(G_{n}\right)\right\} .
$$

This completes the induction.

Theorem 3. Let $\left(a_{i}\right)_{i=1}^{2 n}$ be a strictly decreasing sequence of positive real numbers such that

$$
G_{n}=\bigcup_{i=1}^{n}\left(a_{2 i}, a_{2 i-1}\right]
$$

is a good set, and

$$
\bigcup_{i=1}^{n-1}\left(a_{2 i+1}, a_{2 i}\right] \subseteq \operatorname{Bad}\left(G_{n}\right) .
$$

If $A_{1}$ and $A_{2}$ are positive integers such that $a_{1}=1 / A_{1}$ and $a_{2}=1 / A_{2}$, then there is a strictly increasing sequence $\left(A_{i}\right)_{i=1}^{2 n}$ of positive integers such that

$$
a_{i}=\frac{1}{A_{i}}
$$

for $i=1, \ldots, 2 n$.

Proof. The proof is by induction on $i$. Let $2 \leq i \leq n$ and assume that there are positive integers $A_{1}<\cdots<A_{2 i-2}$ such that $a_{j}=1 / A_{j}$ for $j=1, \ldots, 2 i-2$. We shall prove that there are positive integers $A_{2 i-1}$ and $A_{2 i}$ such that $a_{2 i-1}=1 / A_{2 i-1}$ and $a_{2 i}=1 / A_{2 i}$.

Consider the good number $a_{2 i-1}$. If $h \in \mathbf{N}$ and $h \geq\left(a_{2 i-2}-a_{2 i-1}\right)^{-1}$, then

$$
a_{2 i-1}+\frac{1}{h} \in\left(a_{2 i-1}, a_{2 i-2}\right] \subseteq \operatorname{Bad}\left(G_{n}\right)
$$


and so there exists $r_{h} \in \mathbf{N}^{\sharp}$ such that, for all $j \in\{1,2, \ldots, k-1\}$,

$$
\left(a_{2 i-1}+\frac{1}{h}\right) r_{h}^{j} \in G_{n}
$$

and

$$
a_{2 i-1}<a_{2 i-1} r_{h} \leq a_{2 i-1} r_{h}^{j}<\left(a_{2 i-1}+\frac{1}{h}\right) r_{h}^{k-1} \leq a_{1} .
$$

Therefore,

$$
2 \leq r_{h}<\frac{a_{1}}{a_{2 i-1}} .
$$

Because $a_{2 i-1} \in G_{n}$, there exists $j_{h} \in\{1,2, \ldots, k-1\}$ such that

$$
a_{2 i-1} r_{h}^{j_{h}} \notin G_{n} .
$$

There are only finitely many choices for $r_{h}$ and $j_{h}$. By the pigeonhole principle, there are integers $r \in \mathbf{N}^{\sharp}$ and $j \in\{1,2, \ldots, k-1\}$ and there is a strictly increasing infinite sequence $\left(h_{\ell}\right)_{\ell \in \mathbf{N}}$ of positive integers such that

$$
r_{h_{\ell}}=r \quad \text { and } \quad j_{h_{\ell}}=j
$$

for all $\ell \in \mathbf{N}$. Because $a_{2 i-1} r^{j} \notin G_{n}$ and $a_{2 i-1}<a_{2 i-1} r^{j}<a_{1}$, there is a unique positive integer $t \leq i$ such that $a_{2 i-1} r^{j} \in\left(a_{2 t-1}, a_{2 t-2}\right]$. Because $\left(a_{2 i-1}+1 / h_{\ell}\right) r^{j} \in$ $G_{n}$, it follows that

or, equivalently,

$$
a_{2 i-1} r^{j} \leq a_{2 t-2}<\left(a_{2 i-1}+\frac{1}{h_{\ell}}\right) r^{j}
$$

$$
\frac{a_{2 t-2}}{r^{j}}-\frac{1}{h_{\ell}}<a_{2 i-1} \leq \frac{a_{2 t-2}}{r^{j}} .
$$

By the induction hypothesis, there is a positive integer $A_{2 h-2}$ such that $a_{2 t-2}=$ $1 / A_{2 t-2}$. Letting $\ell \rightarrow \infty$, we obtain

$$
a_{2 i-1}=\frac{a_{2 t-2}}{r^{j}}=\frac{1}{r^{j} A_{2 t-2}}=\frac{1}{A_{2 i-1}}
$$

with $A_{2 i-1}=r^{j} A_{2 t-2}$.

Next we consider the bad number $a_{2 i}$. There exists $r \in \mathbf{N}^{\sharp}$ such that $a_{2 i} r^{j} \in G_{n}$ for all $j \in\{1,2, \ldots, k-1\}$. If $h \geq\left(a_{2 i-1}-a_{2 i}\right)^{-1}$, then

$$
a_{2 i}+\frac{1}{h} \in\left(a_{2 i}, a_{2 i-1}\right] \subseteq G_{n}
$$

and so there exists $j_{h} \in\{1,2, \ldots, k-1\}$ such that

$$
\left(a_{2 i}+\frac{1}{h}\right) r^{j_{h}} \notin G_{n} .
$$

By the pigeonhole principle, there is an integer $j \in\{1,2, \ldots, k-1\}$ and there is a strictly increasing infinite sequence $\left(h_{\ell}\right)_{\ell \in \mathbf{N}}$ of positive integers such that $j_{h_{\ell}}=j$ for all $\ell \in \mathbf{N}$. Because $a_{2 i} r^{j} \in G_{n}$, there is a unique positive integer $t \leq i$ such that $a_{2 i} r^{j} \in\left(a_{2 t}, a_{2 t-1}\right]$. Because $\left(a_{2 i}+1 / h_{\ell}\right) r^{j} \notin G_{n}$, it follows that, for all $\ell \in \mathbf{N}$, we have

or, equivalently,

$$
a_{2 i} r^{j} \leq a_{2 t-1}<\left(a_{2 i}+\frac{1}{h_{\ell}}\right) r^{j}
$$

$$
\frac{a_{2 t-1}}{r^{j}}-\frac{1}{h_{\ell}}<a_{2 i} \leq \frac{a_{2 t-1}}{r^{j}}
$$


By the induction hypothesis, there is a positive integer $A_{2 t-1}$ such that $a_{2 t-1}=$ $1 / A_{2 t-1}$. Letting $\ell \rightarrow \infty$, we obtain

$$
a_{2 i}=\frac{a_{2 t-1}}{r^{j}}=\frac{1}{r^{j} A_{2 t-1}}=\frac{1}{A_{2 i}}
$$

with $A_{2 i}=r^{j} A_{2 t-1}$. This completes the proof.

Theorem 4. Let $\left(a_{i}\right)_{i \in \mathbf{N}}$ be a strictly decreasing infinite sequence of positive real numbers such that

$$
G=\bigcup_{i=1}^{\infty}\left(a_{2 i}, a_{2 i-1}\right]
$$

is a good set, and

$$
\operatorname{Bad}(G)=\bigcup_{i=1}^{\infty}\left(a_{2 i+1}, a_{2 i}\right] .
$$

If $A_{1}$ and $A_{2}$ are positive integers such that $a_{1}=1 / A_{1}$ and $a_{2}=1 / A_{2}$, then there is a strictly increasing infinite sequence $\left(A_{i}\right)_{i \in \mathbf{N}}$ of positive integers such that

$$
a_{i}=\frac{1}{A_{i}}
$$

for all $i \in \mathbf{N}$. Moreover,

$$
\lim _{i \rightarrow \infty} a_{i}=0 .
$$

Proof. Apply Theorem 3 to the good set $G_{n}=\bigcup_{i=1}^{n}\left(a_{2 i}, a_{2 i-1}\right]$.

Because there is a strict increasing sequence $\left(A_{i}\right)_{i=1}^{\infty}$ of positive integers such that $a_{i}=1 / A_{i}$, it follows that

$$
\lim _{i \rightarrow \infty} a_{i}=\lim _{i \rightarrow \infty} \frac{1}{A_{i}}=0 .
$$

This completes the proof.

Theorem 5. Let $k \geq 3$. There exists a unique strictly increasing sequence $\left(A_{i}^{(k)}\right)_{i=1}^{\infty}$ of positive integers with $A_{1}^{(k)}=1$ such that

$$
G^{(k)}=\bigcup_{i=1}^{\infty}\left(\frac{1}{A_{2 i}^{(k)}}, \frac{1}{A_{2 i-1}^{(k)}}\right]
$$

is a $k$-good set and

$$
\operatorname{Bad}\left(G^{(k)}\right)=\bigcup_{i=1}^{\infty}\left(\frac{1}{A_{2 i+1}^{(k)}}, \frac{1}{A_{2 i}^{(k)}}\right]
$$

Proof. The existence and uniqueness of the sequence $\left(A_{i}^{(k)}\right)_{i=1}^{\infty}$ follows immediately from Theorems 2 and 4 .

Note that

$$
\inf G^{(k)}=\inf \operatorname{Bad}\left(G^{(k)}\right)=0
$$

because $\lim _{i \rightarrow \infty} A_{i}^{(k)}=\infty$. 
We have already proved that $A_{2}^{(k)}=2^{k-1}$. We can also determine the integers $A_{3}^{(k)}$ and $A_{4}^{(k)}$. The proofs use a simple arithmetic inequality: If $k \geq 3$, then

$$
\frac{3^{k-1}}{2^{k}}=\frac{1}{2}\left(\frac{3}{2}\right)^{k-1} \geq \frac{1}{2}\left(\frac{3}{2}\right)^{2}=\frac{9}{8}>1
$$

and so $2^{k}<3^{k-1}$. Note that between two consecutive integral powers of 2 there is at most one integral power of 3 .

Theorem 6. If $k \geq 3$, then

$$
A_{3}^{(k)}=2^{k-1}
$$

Proof. Let $G_{1}^{(k)}=\left(1 / 2^{k-1}, 1\right]$. If

$$
\frac{1}{2^{k}}<x \leq \frac{1}{2^{k-1}}
$$

then

$$
\frac{1}{2^{k-1}}=\frac{2}{2^{k}}<2 x<2^{2} x<\cdots<2^{k-1} x \leq \frac{2^{k-1}}{2^{k-1}}=1
$$

and so $\left\{2^{i} x: i=1,2, \ldots, k-1\right\} \subseteq G_{1}^{(k)}$, that is, $x$ is $k$-bad with respect to $G_{1}^{(k)}$.

If $x=1 / 2^{k}$, then $2 x=1 / 2^{k-1} \notin G_{1}^{(k)}$. If $r \geq 3$, then

$$
r^{k-1} x \geq \frac{3^{k-1}}{2^{k}}>1
$$

and so $r^{k-1} x \notin G_{1}^{(k)}$. Therefore, $1 / 2^{k}$ is $k$-good with respect to $G_{1}^{(k)}$, and $A_{3}^{(k)}=$ $2^{k}$.

Theorem 7. Let $k \geq 3$. If there is no integral power of 3 between $2^{k-1}$ and $2^{k}$, then

$$
A_{4}^{(k)}=3^{k-1}
$$

If there is an integral power of 3 between $2^{k-1}$ and $2^{k}$, and if $\ell$ is the positive integer such that

$$
2^{k-1}<3^{\ell}<2^{k}
$$

then $2 \leq \ell \leq k-2$ and

$$
A_{4}^{(k)}=2^{k} 3^{k-1-\ell}=3^{k-1}\left(\frac{2^{k}}{3^{\ell}}\right) .
$$

Inequality (3) is equivalent to $1<2^{k} / 3^{\ell}<2$.

For positive integers $k$, the following are equivalent:

(i) There is an integral power of 3 between $2^{k-1}$ and $2^{k}$.

(ii) The fractional part of $k \log _{3} 2$ is less than $\log _{3} 2$.

(iii) $k$ is in the set $\left\{\left[\ell \log _{2} 3\right]+1: \ell=1,2, \ldots\right\}$. Thus, the formula for $A_{4}^{(k)}$ depends on diophantine properties of logarithms.

Proof. We have $A_{3}^{(k)}=1 / 2^{k}$ by Theorem 6 , Let

For $r \geq 4$ we have

$$
\frac{1}{4^{k-1}}<x \leq \frac{1}{2^{k}}
$$

$$
x r^{k-1} \geq x 4^{k-1}>\frac{4^{k-1}}{4^{k-1}}=1
$$


and so $\left\{x r^{i}: i=1,2, \ldots, k-1\right\} \not \subset G_{1}^{(k)}$.

With $r=2$ we have

$$
x 2^{k-1}>\frac{2^{k-1}}{4^{k-1}}=\frac{1}{2^{k-1}} .
$$

Let $j$ be the smallest integer such that $x 2^{j}>1 / 2^{k-1}$. Then $j \leq k-1$. Because $2 x \leq 2 / 2^{k}=1 / 2^{k-1}$, it follows that $j \geq 2$. If

$$
x 2^{j-1} \leq \frac{1}{2^{k}}
$$

then

$$
x 2^{j} \leq \frac{1}{2^{k-1}}<x 2^{j}
$$

which is absurd. Therefore,

$$
\frac{1}{2^{k}}<x 2^{j-1} \leq \frac{1}{2^{k-1}}
$$

and and so $\left\{x 2^{i}: i=1,2, \ldots, k-1\right\} \not \subset G_{1}^{(k)}$.

The remaining case is the ratio $r=3$ and the geometric progression $\left\{x 3^{i}: i=\right.$ $1,2, \ldots, k-1\}$. If $x>1 / 3^{k-1}$, then $x 3^{k-1}>1$ and $x$ is good with respect to $G_{1}^{(k)}$. Therefore, $A_{4}^{(k)} \geq 3^{k-1}$.

Let $x=1 / 3^{k-1}$. If there exists $j \in\{1,2, \ldots, k-1\}$ such that

$$
\frac{1}{2^{k}}<x 3^{j}=\frac{3^{j}}{3^{k-1}}<\frac{1}{2^{k-1}}
$$

then

$$
2^{k-1}<3^{k-1-j}<2^{k} .
$$

Thus, if there is no power of 3 between $2^{k-1}$ and $2^{k}$, then for all $i \in\{1,2, \ldots, k-1\}$, either $x<3^{i} x<1 / 2^{k}$ or $1 / 2^{k-1}<3^{i} x \leq 1$. Thus, $1 / 3^{k-1}$ is $k$-bad, and $A_{4}^{(k)}=$ $3^{k-1}$.

Suppose that there is a power of 3 between $2^{k-1}$ and $2^{k}$, and that $\ell$ is the unique positive integer that satisfies (3). We observe that $k \geq 4$ because there is no power of 3 between $2^{2}=4$ and $2^{3}=8$, and that $2 \leq \ell \leq k-2$ because $2^{3}<3^{2} \leq 3^{\ell}$ and $2^{k-1}<3^{\ell} \leq 3^{k-2}$. Let

$$
j=k-1-\ell .
$$

Then $1 \leq j \leq k-3$. For $k \geq 4$ we have

$$
\left(\frac{4}{3}\right)^{k-1} \geq\left(\frac{4}{3}\right)^{3}>2
$$

and so

$$
\left(\frac{2}{3}\right)^{k-1}>\frac{1}{2^{k-2}}
$$

Let

$$
x_{0}=\frac{1}{2^{k} 3^{j}}=\frac{3^{\ell}}{2^{k} 3^{k-1}}>\frac{2^{k-1}}{2^{k} 3^{k-1}}=\frac{1}{2^{k}}\left(\frac{2}{3}\right)^{k-1}>\frac{1}{4^{k-1}} .
$$

If

$$
x_{0}<x \leq \frac{1}{3^{k-1}}
$$

then

$$
\frac{1}{2^{k}}=x_{0} 3^{j}<x 3^{j} \leq \frac{3^{j}}{3^{k-1}}=\frac{1}{3^{\ell}}<\frac{1}{2^{k-1}}
$$


and so $x$ is good with respect to $G_{1}^{(k)}$.

It remains only to prove that $x_{0}$ is bad. If $1 \leq i \leq j$, then

$$
x_{0}<x_{0} 3^{i} \leq x_{0} 3^{j}=\frac{1}{2^{k}} .
$$

If $j+1 \leq i \leq k-1$, then

$$
\frac{1}{2^{k-1}}<\frac{3}{2^{k}}=3^{j+1} x_{0} \leq 3^{i} x_{0} \leq 3^{k-1} x_{0}=\frac{3^{\ell}}{2^{k}}<1 .
$$

Thus, $x_{0}=1 /\left(2^{k} 3^{j}\right)$ is bad and $A_{4}^{(k)}=2^{k} 3^{j}$. This completes the proof.

\section{INTEGER SEQUENCES WITH NO $k$-TERM GEOMETRIC PROGRESSION}

If $a$ and $b$ are real numbers with $a \leq b$, then the number of integers in the interval $(a, b]$ is $b-a+\theta$ with $|\theta|<1$.

Recall that, for positive integers $k$ and $n$, the arithmetic function $g_{k}(n)$ denotes the cardinality of the largest subset of the set $\{1,2,3, \ldots, n\}$ that contains no integer geometric progression of length $k$ with integer ratio.

Theorem 8. Let $k \geq 3$, and let $\left(A_{i}^{(k)}\right)_{i=1}^{\infty}$ be the strictly increasing sequence of positive integers constructed in Theorem $\stackrel{i=1}{5}$, Then

$$
\gamma_{k}=\liminf _{n \rightarrow \infty} \frac{g_{k}(n)}{n} \geq \sum_{i=1}^{\infty}\left(\frac{1}{A_{2 i-1}^{(k)}}-\frac{1}{A_{2 i}^{(k)}}\right) .
$$

In particular,

$$
\gamma_{k} \geq 1-\frac{1}{2^{k}}-\frac{1}{3^{k}}
$$

Proof. For every positive integer $h$, the set

$$
G_{h}^{(k)}=\bigcup_{i=1}^{h}\left(\frac{1}{A_{2 i}^{(k)}}, \frac{1}{A_{2 i-1}^{(k)}}\right]
$$

is a $k$-good subset of $(0,1]$. For every positive integer $n$, the dilated set

$$
n * G_{h}^{(k)}=n * \bigcup_{i=1}^{h}\left(\frac{1}{A_{2 i}}, \frac{1}{A_{2 i-1}}\right]=\bigcup_{i=1}^{h}\left(\frac{n}{A_{2 i}}, \frac{n}{A_{2 i-1}}\right]
$$

is a disjoint union of intervals, and so

$$
\begin{aligned}
\left|\left(n * G_{h}^{(k)}\right) \cap \mathbf{N}\right| & =\sum_{i=1}^{h}\left|\left(\frac{n}{A_{2 i}}, \frac{n}{A_{2 i-1}}\right] \cap \mathbf{N}\right| \\
& =n \sum_{i=1}^{h}\left(\frac{1}{A_{2 i-1}}-\frac{1}{A_{2 i}}\right)+\theta_{h}
\end{aligned}
$$

with $\left|\theta_{h}\right|<h$. Because the dilation of a $k$-good set is $k$-good, and a subset of a $k$-good set is $k$-good, it follows that $\left(n * G_{h}^{(k)}\right) \cap \mathbf{N}$ is a $k$-good set of positive integers. Moreover, $A_{1}=1$ implies that $\left(n * G_{h}^{(k)}\right) \cap \mathbf{N}$ is a subset of $\{1,2, \ldots, n\}$. Therefore,

$$
\left|\left(n * G_{h}^{(k)}\right) \cap \mathbf{N}\right| \leq g_{k}(n)
$$


and so

$$
\sum_{i=1}^{h}\left(\frac{1}{A_{2 i-1}}-\frac{1}{A_{2 i}}\right)=\lim _{n \rightarrow \infty} \frac{\left|\left(n * G_{h}^{(k)}\right) \cap \mathbf{N}\right|}{n} \leq \liminf _{n \rightarrow \infty} \frac{g_{k}(n)}{n} .
$$

This inequality holds for all $h \in \mathbf{N}$, and so

$$
\sum_{i=1}^{\infty}\left(\frac{1}{A_{2 i-1}}-\frac{1}{A_{2 i}}\right) \leq \liminf _{n \rightarrow \infty} \frac{g_{k}(n)}{n}=\gamma_{k} .
$$

Applying inequality (4) with $h=2$ and the values for $A_{3}^{(k)}$ and $A_{4}^{(k)}$ computed in Theorems 6] and 7, we obtain

$$
\gamma_{k} \geq\left(1-\frac{1}{2^{k-1}}\right)+\left(\frac{1}{2^{k}}-\frac{1}{3^{k}}\right)=1-\frac{1}{2^{k}}-\frac{1}{3^{k}} .
$$

This completes the proof.

It is a finite calculation to determine explicit values of the integers $A_{i}^{(k)}$ for small values of $i$ and $k$. Table 1 contains all values of $A_{i}^{(k)}$ for $3 \leq k \leq 9$ with $A_{i}^{(k)}<10^{6}$. Applying inequality (4) in Theorem [8, we can use these values to get lower bounds for $\gamma_{k}$ that improve results obtained previously by Rankin [6] and Riddell [7]. For $k=3$, McNew [3] has the current best lower bound. Related results have been obtained by Brown and Gordon [2], Beiglböck, Bergelson, Hindman, and Strauss [1, and Nathanson and O'Bryant [4, 5].

The following table records upper and lower bounds for $\gamma_{k}$.

\begin{tabular}{|c|cccc|c|ccc|}
\hline & \multicolumn{5}{|c|}{ Lower bounds on $\gamma_{k}$} & & \multicolumn{3}{|c|}{ Upper bounds on $\gamma_{k}$} \\
$k$ & Rankin & Riddell & This paper & McNew & $\mathrm{k}$ & McNew & From $r_{k}$ & Riddell \\
\hline 3 & 0.719745 & & 0.815870 & 0.818410 & 3 & 0.819222 & 0.846376 & 0.857143 \\
4 & 0.862601 & 0.895283 & 0.919818 & & 4 & & 0.928874 & 0.933334 \\
5 & 0.931652 & 0.958056 & 0.963737 & & 5 & & 0.967742 & 0.967742 \\
6 & 0.966324 & 0.980371 & 0.982877 & & 6 & & 0.983871 & 0.984126 \\
7 & 0.983438 & 0.991159 & 0.991805 & & 7 & & & 0.992126 \\
8 & 0.991841 & 0.995717 & 0.995913 & & 8 & & & 0.996079 \\
9 & 0.995969 & 0.997939 & 0.998012 & & 9 & & & 0.998044 \\
\hline
\end{tabular}

\section{REFERENCES}

[1] M. Beiglböck, V. Bergelson, N. Hindman, and D. Strauss, Multiplicative structures in additively large sets, J. Combin. Theory Ser. A 113 (2006), no. 7, 1219-1242.

[2] B. E. Brown and D. M. Gordon, On sequences without geometric progressions, Math. Comp. 65 (1996), no. 216, 1749-1754.

[3] N. McNew, On sets of integers which contain no three terms in geometric progression, arXiv: 1310.2277, 2013.

[4] M. B. Nathanson and K. O'Bryant, On sequences without geometric progressions, Integers 13 (2013), \#A73, 1-5.

[5] Irrational numbers associated to sequences without geometric progressions, Integers 14 (2014).

[6] R. A. Rankin, Sets of integers containing not more than a given number of terms in arithmetical progression, Proc. Roy. Soc. Edinburgh Sect. A 65 (1960/1961), 332-344 (1960/61).

[7] J. Riddell, Sets of integers containing no $n$ terms in geometric progression, Glasgow Math. J. 10 (1969), 137-146. 


\begin{tabular}{|c|c|c|c|c|c|c|c|}
\hline$i$ & 3 & 4 & 5 & $\begin{array}{l}k \\
6\end{array}$ & 7 & 8 & 9 \\
\hline 1 & 1 & 1 & 1 & 1 & 1 & 1 & 1 \\
\hline 2 & 4 & 8 & 16 & 32 & 64 & 128 & 256 \\
\hline 3 & 8 & 16 & 32 & 64 & 128 & 256 & 512 \\
\hline 4 & 9 & 48 & 96 & 243 & 1152 & 2304 & 6561 \\
\hline 5 & 12 & 200 & 144 & 288 & 1728 & 3456 & 6912 \\
\hline 6 & 24 & 216 & 576 & 576 & 8192 & 16384 & 13824 \\
\hline 7 & 27 & 288 & 4032 & 729 & 28800 & 32768 & 19683 \\
\hline 8 & 32 & 1200 & 4096 & 1152 & 172800 & 163840 & 131072 \\
\hline 9 & 36 & 1296 & 4608 & 2048 & 248832 & 288000 & 221184 \\
\hline 10 & 40 & 1400 & 32256 & 3645 & 307328 & 331776 & 492075 \\
\hline 11 & 45 & 1512 & 32768 & 4000 & 395136 & 497664 & 655360 \\
\hline 12 & 48 & 1600 & 36288 & 10240 & & 884736 & \\
\hline 13 & 2208 & 1728 & 36864 & 20736 & & 995328 & \\
\hline 14 & 2209 & 1800 & 40320 & 21952 & & & \\
\hline 15 & 2256 & 1944 & 40960 & 92160 & & & \\
\hline 16 & 8832 & 2000 & 41472 & 100000 & & & \\
\hline 17 & 8836 & 62400 & 129600 & 102400 & & & \\
\hline 18 & 9024 & 63936 & 131072 & 207360 & & & \\
\hline 19 & 17664 & 73800 & 147456 & 219520 & & & \\
\hline 20 & 17672 & 74088 & 157216 & 518400 & & & \\
\hline 21 & 18048 & 75600 & 166464 & 548800 & & & \\
\hline 22 & 19872 & 79704 & & 921600 & & & \\
\hline 23 & 19881 & 80688 & & & & & \\
\hline 24 & 20304 & 81648 & & & & & \\
\hline 25 & 26496 & 88000 & & & & & \\
\hline 26 & 26508 & 499200 & & & & & \\
\hline 27 & 27072 & 511488 & & & & & \\
\hline 28 & 52992 & 590400 & & & & & \\
\hline 29 & 53016 & 592704 & & & & & \\
\hline 30 & 54144 & 604800 & & & & & \\
\hline 31 & 59616 & 637632 & & & & & \\
\hline 32 & 59643 & 645504 & & & & & \\
\hline 33 & 60912 & 653184 & & & & & \\
\hline 34 & 70656 & 704000 & & & & & \\
\hline 35 & 70688 & 998400 & & & & & \\
\hline 36 & 72192 & & & & & & \\
\hline 37 & 79488 & & & & & & \\
\hline 38 & 79524 & & & & & & \\
\hline 39 & 81216 & & & & & & \\
\hline 40 & 88320 & & & & & & \\
\hline 41 & 88360 & & & & & & \\
\hline 42 & 90240 & & & & & & \\
\hline 43 & 99360 & & & & & & \\
\hline 44 & 99405 & & & & & & \\
\hline 45 & 101520 & & & & & & \\
\hline 46 & 103776 & & & & & & \\
\hline 47 & 103823 & & & & & & \\
\hline 48 & 105984 & & & & & & \\
\hline 49 & 106032 & & & & & & \\
\hline 50 & 108192 & & & & & & \\
\hline 51 & 108241 & & & & & & \\
\hline 52 & 108288 & & & & & & \\
\hline
\end{tabular}

TABLE 1 . For $3 \leq k \leq 9$, the table contains all integers $A_{i}^{(k)}$ satisfying Theorem 5 that are less than $10^{6}$. These numbers are sequences A235054-60 in the OEIS. 
Department of Mathematics, Lehman College (CUNy), Bronx, NY 10468

E-mail address: melvyn.nathanson@lehman.cuny.edu

Department of Mathematics, College of Staten Island (CUNy), Staten Island, Ny 10314

E-mail address: kevin@member.ams.org 